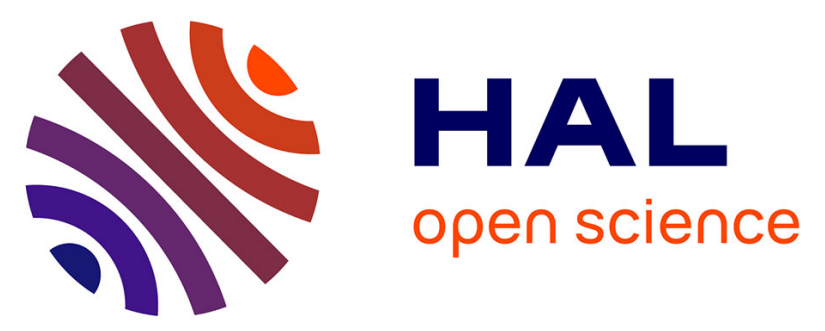

\title{
Comparaison of two homogenization methods using a damage model for a fibrous membrane, based on the fibers' fracture process at the microscale.
}

\author{
Aline Brunon, Michel Coret, Karine Bruyere-Garnier, Alain Combescure
}

\section{To cite this version:}

Aline Brunon, Michel Coret, Karine Bruyere-Garnier, Alain Combescure. Comparaison of two homogenization methods using a damage model for a fibrous membrane, based on the fibers' fracture process at the microscale.. European Journal of Mechanics - A/Solids, 2013, 39, pp.1-10. 10.1016/j.euromechsol.2012.10.006 . hal-00789316v2

\section{HAL Id: hal-00789316 \\ https://hal.science/hal-00789316v2}

Submitted on 29 May 2018

HAL is a multi-disciplinary open access archive for the deposit and dissemination of scientific research documents, whether they are published or not. The documents may come from teaching and research institutions in France or abroad, or from public or private research centers.
L'archive ouverte pluridisciplinaire HAL, est destinée au dépôt et à la diffusion de documents scientifiques de niveau recherche, publiés ou non, émanant des établissements d'enseignement et de recherche français ou étrangers, des laboratoires publics ou privés. 


\title{
Comparaison of two homogenization methods using a damage model for a fibrous membrane, based on the fibers' fracture process at the microscale.
}

\author{
A. Bel-Brunon ${ }^{\mathrm{a}, \mathrm{b}}$, M. Coret $^{\mathrm{b}}$, K. Bruyère-Garnier ${ }^{\mathrm{a}}$, A. Combescure ${ }^{*, \mathrm{~b}}$ \\ Université de Lyon, Lyon, F-69000, France \\ ${ }^{a}$ Ifsttar, UMR_T9406, LBMC, F-69675, Bron, France \\ Université Lyon 1, F-69622, Villeurbanne, France \\ ${ }^{b}$ INSA-Lyon, LaMCoS UMR5259,F-69621, France
}

\begin{abstract}
The objective of this paper is to evaluate two existing homogenization methods using a simple damage model for fibrous membrane and compare them. A macroscopic damage model of a fibrous membrane tissue based on the knowledge of the behavior and fracture process of a single fiber is constructed. Under some simplifying assumptions (linear behavior of the fibers, brittle fibers, constant angular distribution of the fibers), the evolution of fiber fracture is described by two macroscopic scalar variables; this enables the tissue's damage to be modeled without requiring a second discretization at the microscale. For the homogenization of the fibers contribution, an energetic method and a kinematic method are adapted and compared. Both lead to similar macroscopic behavior in the elastic phase, but the behavior differs in the fracture
\end{abstract}

\footnotetext{
*Corresponding author. Laboratoire de Mécanique des Contacts et des Structures Institut National des Sciences Appliquées de Lyon (INSA), Bâtiment Jean d'Alembert, 18-20 rue des Sciences, F69621 Villeurbanne Cedex, France. Tel:+33472436426; Fax:+33478890980.

Email address: alain.combescure@insa-lyon.fr (A. Combescure)
} 
phase of the homogenized material. In the case of biological materials, the proposed law enables taking into account the often observed phenomena of crimping and damage of the fibers.

Key words: fibrous membrane tissue, damage, microstructure, homogenization, biaxial tension

\section{Introduction}

The question of the fracture modeling of fibrous tissues arises in many domains, including composites, papers and biological tissues. In the latter case, the large presence of fibrous tissues of different natures in the human body lead to an general research effort to explain how the macroscopic behavior of those tissues is related to their microstructure, especially in the case of non-homogeneous fiber distributions. In terms of modeling, two aspects have to be considered : 1) the fibrous nature of the tissue and 2) the evolution of damage in the tissue. Several options are proposed in the literature to assess the first point. Using invariants to take into account the isotropic or anisotropic nature of the fibrous contribution is a first option, introduced in (Holzapfel, 2000). This method does not require any further homogenization as the invariants already are a macroscopic representation of the fiber orientations and assumes that the fibers are perfectly aligned along one or several directions. If the fibers are distributed around one or several preferred directions, a statistical distribution of the fibers is generally introduced. From there, two options are suggested in the literature: either a structure tensor (Gasser et al., 2006) can be used to provide a macroscopic representation of the microstructure (the kinematic method) or the individual energetic con- 
tributions of the fibers are summed up (Lanir, 1983) (the energetic method). A more recent option is the microsphere-based approach (Menzel and Waffenschmidt, 2009; Sáez et al., 2012) which uses spherical units in which the homogenization process is conducted. The anisotropy of the tissue is then described by associating different weights to the fiber directions. Using a limited number of integration directions was proved to be enough to provide good accuracy as well as a low computational cost to the two-scale simulation of 3D tissues. Another recent feature is the macromolecular network model proposed by (Kuhl et al., 2005), based on eight-chain unit cells and which can handle fiber reorientation.

In this paper, the attention is focused on how the fiber distribution is taken into account: both the kinematic and energetic methods are adapted to the same fibrous tissue model to assess whether and in which domain they are equivalent.

The second point in modeling the fracture of fibrous tissue is the description of the damage. In the field of biomechanics, the problem of the fracture of fibrous tissues has received only limited attention. Some authors model damage continuously using an internal damage variable which is to be identified for 1D (Calvo et al., 2007) or 2D tissues (Balzani et al., 2006). (Rodríguez et al., 2006) introduces a 3D model which also describes damage continuously, but uses two independent variables for the matrix and the fibers. Other papers describe damage in the tissue as the result of fiber fracture on the lower scale: (Hurschler et al., 1997) proposes a tendon-andligament model involving three scales (the fibrils', the fibers' and the tissue's) and studies the behavior and damage of the tissue in the case of fibers 
aligned with a single direction. (Liao and Belkoff, 1999) also proposes a fracture model of a 1D tissue, but takes into account the initial crimping of the fibers. (Gasser and Holzapfel, 2006) models the fracture of arteries by dissection, i.e. splitting through the thickness, which constitutes a different fracture mode than surface tearing. (Cacho et al., 2007) proposes a damage model for a fibrous tissue made of initially crimped fibers. More recently, (Sáez et al., 2012) introduced damage in a microsphere model of the blood vessel taking into account its initial anisotropy.

The fibrous tissue modeling in the present paper is similar to that from (Cacho et al., 2007). The idea of our model is to describe the evolution of the macroscopic damage in a fibrous membrane and therefore of the tissue anisotropy during a biaxial strain loading; under simplifying assumptions, it will be shown that two scalar variables are sufficient for this purpose. This simple model, including some extensions related to biological tissues, is used to illustrate the comparison of the two homogenization methods.

The outline of this paper is the following: the first part presents the two homogenization methods adapted to a fibrous membrane model; the second part is focused on the description of the simple damage model that we propose; the third part illustrates the comparison of the two homogenization methods using our damage model; the fourth part presents extensions of the damage model to biological phenomena such as damageable fibers and fiber crimp, as well as the consequences on the homogenization methods. 


\section{Modeling the tissue fracture}

Let us consider a fibrous membrane and suppose that we know the influence of a biaxial tension loading on the fibers breakage. We can proceed to homogenize the behavior. Several homogenization methods are available in the literature; the most commonly used are the ones described in (Lanir, 1983) and (Gasser et al., 2006). The first one consists in an energetic homogenization of the fibers while the second is based on a kinematic homogenization. In this paper, these two methods are adapted to the fibrous tissue damage problem and compared.

\subsection{General framework}

This section takes up the same general framework as in (Gasser et al., 2006). We consider a plane tissue consisting of a matrix and fibers. The free energy $\psi$ of the tissue is the sum of the free energy of the matrix $\psi_{m}$ and the free energy of the fibers $\psi_{f}$. A common way to enforce compressibility/incompressibility conditions is to split the strain energy of the matrix into a volumetric part $U_{m}$ and an isochoric part $\bar{\psi}_{m}$. It is usually assumed that the incompressibility of biological tissues is due to the water content of the ground matrix and that the fibers' contribution is negligible in the volumetric part of the free energy. Therefore, it is not necessary to split the fibers' contribution into volumetric and isochoric parts.

Hence the free energy of the tissue can be written as:

$$
\psi=\psi_{m}+\psi_{f}=U_{m}+\bar{\psi}_{m}+\psi_{f}
$$


First we will address the matrix free energy expression; then, we will examine two homogenization methods for the contribution of the fibers.

\subsection{Contribution of the matrix}

The volumetric/isochoric decomposition is applied to the gradient of transformation $\mathbf{F}$, i.e. to the right Cauchy-Green strain tensor $\mathbf{C}$ :

$$
\begin{aligned}
\mathbf{F} & =J^{\frac{1}{3}} \overline{\mathbf{F}} \\
\mathbf{C} & =J^{\frac{2}{3}} \overline{\mathbf{C}}
\end{aligned}
$$

with $J=\operatorname{det}(\mathbf{F})$. The cumulative energy decomposition enables the second Piola-Kirchhoff stress tensor (PK2) to be expressed as:

$$
\mathbf{S}_{m}=2 \frac{\partial \psi_{m}}{\partial \mathbf{C}}=2\left(\frac{\partial U_{m}}{\partial \mathbf{C}}+\frac{\partial \bar{\psi}_{m}}{\partial \mathbf{C}}\right)=\mathbf{S}_{m}^{v o l}+\overline{\mathbf{S}}_{m}
$$

We set $p=\partial U_{m} / \partial J$; we also assume that the matrix follows an isotropic, neo-Hookean behavior expressed by $\bar{\psi}_{m}=c(\operatorname{tr} \overline{\mathbf{C}}-3) / 2$, where $c$ is a material parameter. Then:

$$
\begin{gathered}
\mathbf{S}_{m}^{v o l}=2 \frac{\partial U_{m}}{\partial \mathbf{C}}=2 \frac{\partial U_{m}}{\partial J} \frac{\partial J}{\partial \mathbf{C}}=p J \mathbf{C}^{-1} \\
\overline{\mathbf{S}}_{\mathbf{m}}=2 \frac{\partial \bar{\psi}_{m}}{\partial \mathbf{C}}=2 \frac{\partial \bar{\psi}_{m}}{\partial \overline{\mathbf{C}}}: \frac{\partial \overline{\mathbf{C}}}{\partial \mathbf{C}}=J^{-\frac{2}{3}} \mathbb{P}: c \mathbf{I}
\end{gathered}
$$

with the fourth-order operators $\mathbb{P}=\mathbb{I}-\frac{1}{3} \mathbf{C}^{-1} \otimes \mathbf{C}$ and $I_{i j k l}=\left(\delta_{i k} \delta_{j l}+\delta_{i l} \delta_{j k}\right) / 2$, where $\delta_{i j}$ is Kronecker's symbol.

Thus, the contribution of the matrix to PK2 tensor becomes:

$$
\mathbf{S}_{m}=p J \mathbf{C}^{-1}+J^{-\frac{2}{3}} \mathbb{P}: c \mathbf{I}
$$




\subsection{Contribution of the fibers}

\subsubsection{The concept of fiber density function}

In order to describe the strain energy in the tissue, we introduce the concept of angular fiber density, denoted $\rho(\xi)$. This function defines the number of the fibers whose orientation belongs to the interval $[\xi, \xi+d \xi]$. Before damage occurs, this function is normalized, i.e.:

$$
\frac{1}{\pi} \int_{-\frac{\pi}{2}}^{\frac{\pi}{2}} \rho(\xi) \mathrm{d} \xi=\frac{1}{\pi} \int_{A_{0}} \rho(\xi) \mathrm{d} \xi=1
$$

In the case of a homogeneous distribution, i.e. $\rho(\xi)=$ constant, $\rho(\xi)=$ $1 \forall \xi \in A_{0}=[-\pi / 2, \pi / 2]$.

Once damage occurs, the density is a function of damage state $\mathbf{D}$. The integration domain evolves and is then denoted $A(\mathbf{D})$. Given this description, we can write :

$$
\int_{A_{0}} \rho(\xi, \mathbf{D}) d \xi=\int_{A(\mathbf{D})} \rho(\xi) d \xi
$$

$A(\mathbf{D})$ represents the domain where the fibers are not broken.

\subsubsection{The Energetic (E) homogenization method}

In this method, the strain energy of the fibrous part of the tissue is assumed to be the sum of the strain energies of the strained, but undamaged, fibers. A fiber subjected to a Green-Lagrange strain field $\mathbf{E}$ is strained only along its longitudinal axis $\mathbf{n}(\xi)$ and its strain energy is $\phi_{f}=\phi_{f}\left(\varepsilon_{f}\right)$ i.e. 
$\phi_{f}(\xi, \mathbf{E})$, whose expression depends on the constitutive relation of the fiber. Therefore, on the tissue's scale, the free energy $\psi_{f}^{E}$ of the fibers is:

$$
\psi_{f}^{E}(\mathbf{E}, \mathbf{D})=\frac{1}{\pi} \int_{A_{0}} \rho(\xi, \mathbf{D}) \phi_{f}(\xi, \mathbf{E}) \mathrm{d} \xi
$$

The expression of stress tensor PK2 is:

$$
\mathbf{S}_{f}^{E}=\frac{\partial \psi_{f}^{E}(\mathbf{E}, \mathbf{D})}{\partial \mathbf{E}}=\frac{1}{\pi} \int_{A_{0}} \rho(\xi, \mathbf{D}) \frac{\partial \phi_{f}(\xi, \mathbf{E})}{\partial \mathbf{E}} \mathrm{d} \xi
$$

Now let us consider the particular case where the fibers behave linearly before damage. The total free energy of the fibrous part of the tissue is:

$$
\psi_{f}^{E}(\mathbf{E}, \mathbf{D})=\frac{1}{\pi} \int_{A_{0}} \rho(\xi, \mathbf{D}) \frac{1}{2} E(\mathbf{M}: \mathbf{E})^{2} \mathrm{~d} \xi
$$

where $E$ is the Young's modulus of the fiber's material and $\mathbf{M}=\mathbf{n}(\xi) \otimes$ $\mathbf{n}(\xi)$ the orientation tensor (see Section 3). Thus, the expression of stress tensor PK2 is:

$$
\mathbf{S}_{f}^{E}=\frac{E}{\pi} \int_{A_{0}} \rho(\xi, \mathbf{D})(\mathbf{M}: \mathbf{E}) \mathbf{M} \mathrm{d} \xi
$$

21 Eq. (11) is valid if we suppose that the variation of the integral of the density function $\int_{A_{0}} \rho(\xi, D)$ with strain is negligible compared to the variation of $\phi_{f}(\xi, \mathbf{E})$, which is the case for the simple model considered in this approach. Therefore only the inner member of the integral is differentiated with respect to $\mathbf{E}$. 


\subsubsection{The Kinematic (K) method}

The kinematic method is derived from works described in (Gasser et al., 2006), among others. We introduce a generalized second-order structure tensor $\mathbf{H}$ defined by Eq.(14). This tensor is used as a macroscopic projector of the strain tensor onto the structure of the undamaged fibers.

$$
\mathbf{H}=\frac{1}{\pi} \int_{A_{0}} \rho(\xi, D) \mathbf{n}(\xi) \otimes \mathbf{n}(\xi) \mathrm{d} \xi
$$

$H_{33}$ equals zero because the fibers are oriented only perpendicularly to Direction 3.

Thus, the constitutive law is applied to the tissue rather than to its constituent fibers, taking the scalar $E_{h}=\mathbf{H}: \mathbf{E}$ as the strain value to express the macroscopic strain energy $\psi_{f}^{K}$ here in the linear case (Eq.15).

$$
\psi_{f}^{K}=\frac{1}{2} E \cdot E_{h}^{2}=\frac{1}{2} E(\mathbf{H}: \mathbf{E})^{2}
$$

From that expression, we deduce the PK2 tensor corresponding to the $K$ method:

$$
\mathbf{S}_{f}^{K}=\frac{\partial \psi_{f}^{K}}{\partial \mathbf{E}}=E(\mathbf{H}: \mathbf{E}) \mathbf{H}
$$

Once again, the damage variable $\mathbf{D}$ and the Green-Lagrange strain tensor $\mathbf{E}$ are independent variables. So $\mathbf{H}$ is not differentiated with respect to $\mathbf{E}$.

\subsection{Association of contributions from the matrix and the fibers}

Let us recall that the total energy of the tissue can be divided into three parts (Eq.1) including two from the matrix contribution (Eq.7). 

of the two forms:

$$
\begin{gathered}
\mathbf{S}^{E}=p J \mathbf{C}^{-1}+J^{-\frac{2}{3}} \mathbb{P}: c \mathbf{I}+\frac{2 E}{\pi} \int_{A_{0}} \rho(\xi, \mathbf{D})(\mathbf{M}: \mathbf{E}) \mathbf{M d} \xi \\
\mathbf{S}^{K}=p J \mathbf{C}^{-1}+J^{-\frac{2}{3}} \mathbb{P}: c \mathbf{I}+E(\mathbf{H}: \mathbf{E}) \mathbf{H}
\end{gathered}
$$

${ }_{143}$ Since the objective is to implement the model into a finite element calcu144 lation code, the tissue is modeled in 3D and plane stresses; in order to apply this condition, we define the Cauchy stress tensor $\mathbf{T}$ :

$$
\mathbf{T}=J^{-1} \mathbf{F} . \mathbf{S} . \mathbf{F}^{T}
$$

The components derived from the matrix contribution are:

$$
\begin{aligned}
\mathbf{T}_{m}^{v o l} & =p \mathbf{I} \\
\overline{\mathbf{T}}_{m} & =J^{-\frac{5}{3}} c\left(\mathbf{B}-\frac{1}{3} \operatorname{tr}\left(\mathbf{C}^{-1}\right) \mathbf{I}\right)
\end{aligned}
$$

${ }_{146}$ where $\mathbf{B}=\mathbf{F} \cdot \mathbf{F}^{T}$ is the left Cauchy-Green tensor. Concerning the contri147 bution of the fibers, we distinguish the two homogenization methods:

$$
\begin{aligned}
& \mathbf{T}_{f}^{E}=J^{-1} \mathbf{F} \cdot \mathbf{S}_{f}^{E} \cdot \mathbf{F}^{T}=\frac{2 E}{J \pi} \mathbf{F} \cdot \int_{A_{0}} \rho(\xi, \mathbf{D})(\mathbf{M}: \mathbf{E}) \mathbf{M} d \xi \cdot \mathbf{F}^{T} \\
& \mathbf{T}_{f}^{K}=J^{-1} \mathbf{F} \cdot \mathbf{S}_{f}^{E} \cdot \mathbf{F}^{T}=\frac{E}{J}(\mathbf{H}: \mathbf{E}) \mathbf{F} \cdot \mathbf{H} \cdot \mathbf{F}^{T}
\end{aligned}
$$


We can notice that the fibrous contribution to the overall tissue does not create a Cauchy stress components out of the plane; this is due to the formulation of the fiber density function and to the fact that we do not split the fibrous contribution into a volumetric component and an isochoric component.

The plane stress condition is expressed by:

$$
T_{33}=p+J^{-\frac{5}{3}} c\left(\lambda_{3}^{2}-\frac{1}{3}\left(\lambda_{1}^{2}+\lambda_{2}^{2}+\lambda_{3}^{2}\right)\right)=0
$$

We can observe that this equation involves $p$, the material characteristics of the matrix and the components $\lambda_{i}$ of the deformation gradient tensor. Here, as is commonly assumed for soft biological tissues, the matrix is considered to be incompressible. In this case, the strain component across the thickness is defined by Eq.(25) and $p$ is a Lagrange multiplier determined by the plane stress condition (Eq.26).

$$
\begin{array}{r}
J=1 \Leftrightarrow \lambda_{3}=\frac{1}{\lambda_{1} \lambda_{2}} \\
p=-\frac{c}{3}\left(\frac{2}{\lambda_{1}^{2} \lambda_{2}^{2}}-\lambda_{1}^{2}-\lambda_{2}^{2}\right)
\end{array}
$$

The two homogenization methods have been described; the next section presents the damage model that was constructed to compare them.

\section{A simple kinematic fracturing fiber model}

In this section, we propose a simple model to describe the evolution of the macroscopic damage of a plane fibrous tissue subjected to biaxial tension 
loading, which is assumed to be the result of fiber breakage on the microscale. The two objectives of this section are first, to build a damage evolution law for the macroscale damage, taking into account its anisotropic nature due to the microscale phenomena; second, to compare the two homogenization methods presented in the previous section.

The underlying assumptions of this section are the following:

- the fibers are rectilinear, linear elastic, and brittle;

- prior to damage, the angular distribution function of the fibers is known, continuous and nonzero;

- the phenomenon of fiber reorientation during loading is neglected ;

- the principal directions of the biaxial strain loading do not change.

The third assumption relies on the conclusions of Sacks and Gloeckner (1999), which observed that the closer the loading to equibiaxiality, the lesser the reorientation of the fibers. Therefore, in the framework of the present study which focuses on biaxial loading, it appears acceptable to ignore fiber reorientation.

This model is academic. It is designed to produce clear conclusions when the two homogenization methods proposed in the previous section are compared. It can be extended using a two scale approach to more realistic situations as uncrimping, damage, non isotropic fiber orientations: the price to pay to these extensions is a larger number of internal variables to describe the small scale state. 
194

\subsection{Parameterization of the problem}

Let us consider a plane fibrous tissue. In the material plane $\left(\mathbf{X}_{\mathbf{m}}, \mathbf{Y}_{\mathbf{m}}, \mathbf{Z}_{\mathbf{m}}\right)$, the direction of a fiber is characterized by the angle $\xi \in[-\pi / 2, \pi / 2]$ and its initial direction vector $\mathbf{n}$ defined by:

$$
\mathbf{n}=\cos \xi \mathbf{X}_{\mathbf{m}}+\sin \xi \mathbf{Y}_{\mathbf{m}}
$$

The tissue is subjected to a biaxial strain characterized by the macroscopic Green-Lagrange strain tensor E described in Cartesian coordinates by:

$$
\begin{aligned}
\mathbf{E} & =\varepsilon_{r} k\left(\cos \varphi \mathbf{X}_{\mathbf{m}} \otimes \mathbf{X}_{\mathbf{m}}+\sin \varphi \mathbf{Y}_{\mathbf{m}} \otimes \mathbf{Y}_{\mathbf{m}}\right) \\
& =E_{1} \mathbf{X}_{\mathbf{m}} \otimes \mathbf{X}_{\mathbf{m}}+E_{2} \mathbf{Y}_{\mathbf{m}} \otimes \mathbf{Y}_{\mathbf{m}}
\end{aligned}
$$

where $\varepsilon_{r}$ is the ultimate longitudinal strain of the fibers. $\varphi$ is the loading angle. From here on, we will assume that $\varphi \in[0, \pi / 2]$ and $k \geq 0$, which implies strict biaxial tension.

The fibers constituting the tissue are uniaxial elements which can withstand only solicitations along their axis. The longitudinal Green strain $\varepsilon_{f}$ of a fiber oriented along an angle $\xi$ is defined by:

$$
\varepsilon_{f}=\mathbf{n}(\xi) \cdot \mathbf{E} \cdot \mathbf{n}(\xi)=k \varepsilon_{r}\left(\cos \varphi \cdot \cos ^{2} \xi+\sin \varphi \sin ^{2} \xi\right)
$$

We can observe that for $\varphi=\frac{\pi}{4}$ all the fibers are solicited equally; then, their longitudinal strain is $\varepsilon_{f}=\frac{k \varepsilon_{r}}{\sqrt{2}}$. Also, differentiating $\varphi$ with respect to 
$\xi$ shows that the most highly loaded fibers are oriented along the principal directions of the strain tensor, that is $\xi=0$ or $\xi=\frac{\pi}{2}$.

\subsection{Initial elasticity range}

The elasticity range $\mathcal{D}$ of a fiber is defined in the strain space by:

$$
\mathcal{D}=\left\{\varepsilon_{f} / F\left(\varepsilon_{f}\right)=\varepsilon_{f}-\varepsilon_{r}<0\right\}
$$

The corresponding elasticity range of the tissue, denoted $\mathcal{S}$, is:

$$
\mathcal{S}=\left\{\mathbf{E} / \forall \xi, \mathbf{n}(\xi) . \mathbf{E} \cdot \mathbf{n}(\xi)-\varepsilon_{r}<0\right\}
$$

The shape of $\mathcal{S}$ corresponds to the resolution of the equation $\varepsilon_{f}-\varepsilon_{r}<0$ and is described by:

$$
\begin{cases}k<\frac{1}{\cos \varphi} & \forall \varphi \in\left[0, \frac{\pi}{4}\right] \\ k<\frac{1}{\sin \varphi} & \forall \varphi \in\left[\frac{\pi}{4}, \frac{\pi}{2}\right]\end{cases}
$$

At the boundary of $\mathcal{S}$, at least one fiber breaks as the non-rupture criterion is not respected anymore (Eq.31). The first fiber to break is always the one oriented along $\xi=0$ if $\varphi \leq \frac{\pi}{4}$ or the one oriented along $\xi=\frac{\pi}{2}$ if $\varphi \geq \frac{\pi}{4}$. The next section describes the damage process of the fibrous tissue.

\subsection{Damage evolution}

We now consider different loading cases to study the evolution of the damage state of this model at the micro scale. Different cases are treated to better understand the potentialities of the model. 
Case of a proportional loading. We assume that the tissue is subjected to a proportional strain loading (i.e. with $\varphi$ constant) so as to reach a point defined by $(k, \varphi)$ such that:

$$
\begin{cases}k>\frac{1}{\cos \varphi} & \text { if } \varphi \in\left[0, \frac{\pi}{4}\right] \\ k>\frac{1}{\sin \varphi} & \text { if } \varphi \in\left[\frac{\pi}{4}, \frac{\pi}{2}\right]\end{cases}
$$

The damaged state at the micro scale is defined by two subsets: the subset of healthy fibers and the set of broken ones. These sets are simply defined by two angles $\xi_{1}$ and $\xi_{2}$. From here on, the vector of the two damage variables $\xi_{1}$ and $\xi_{2}$ will be denoted $\mathbf{D}$ which characterizes the damaged tissue state. These angles are obtained by the solution of equation $\varepsilon_{f}(\xi)-\varepsilon_{r}>0$ (which is detailed in Appendix A). A proportional loading with an intensity $k$ greater than the bounds defined in Eq.(33) leads to the fracture of the fibers as follows:

$$
\begin{aligned}
& \varphi \in\left[0, \frac{\pi}{4}\right]:\left\{\begin{array}{l}
\text { all fibers are broken } \forall \xi \in\left[-\xi_{1}, \xi_{1}\right] \\
\xi_{1}=\arccos \sqrt{\frac{1-k \sin \varphi}{k(\cos \varphi-\sin \varphi)}}
\end{array}\right. \\
& \varphi=\frac{\pi}{4} \text { : all fibers break simultaneously at } k=\sqrt{2} \text { (Eq.30) } \\
& \varphi \in\left[\frac{\pi}{4}, \frac{\pi}{2}\right]:\left\{\begin{array}{l}
\text { all fibers are broken } \forall \xi \in\left[-\frac{\pi}{2},-\xi_{2}\right] \cup\left[\xi_{2}, \frac{\pi}{2}\right] \\
\xi_{2}=\arccos \sqrt{\frac{1-k \sin \varphi}{k(\cos \varphi-\sin \varphi)}}
\end{array}\right.
\end{aligned}
$$

Micro scale damage state and the angles $\xi_{1}$ and $\xi_{2}$ are illustrated in Fig.1 for two typical cases: $\{\varphi=\pi / 6, k=1.7\}$ and $\{\varphi=\pi / 2.7, k=1.2\}$. Fig.2 displays the evolution of $\xi_{1}$ with increasing $k$ values for different angles $\varphi$. 


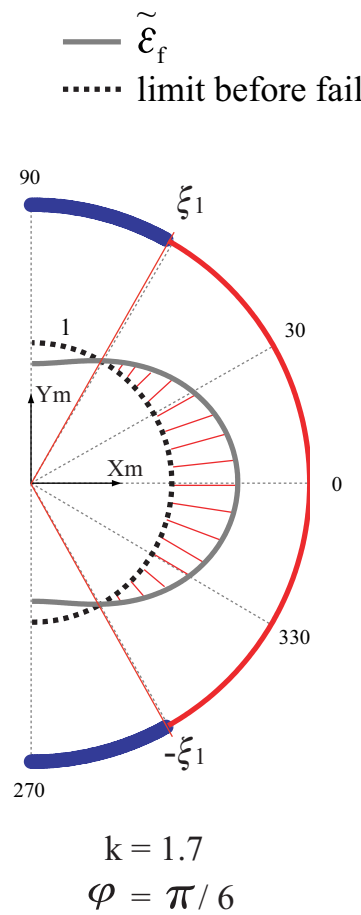

- healthy fibers broken fibers

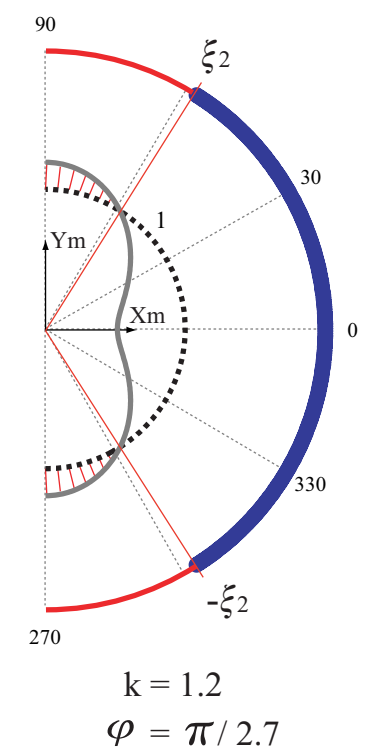

Figure 1: Examples of fiber damage states: if the normalized strain $\tilde{\varepsilon_{f}}=\frac{\varepsilon_{f}}{\varepsilon_{r}}$ is greater than 1 (the red-hatched zones), the fibers break.

Loading case of a constant-amplitude strain but rotating angle $\varphi$. It is interesting to treat the case of a constant-amplitude strain $(k=c t e)$ separately because it corresponds to an evolution of damage that is not monotonic. If $k<\sqrt{2}$ and $\varphi$ evolves between 0 and $\pi / 2$, fiber fracture occurs near $\xi=0$ and $\xi=\pi / 2$, but the fibers near $\pi / 4$ are unaffected (Fig.3 illustrates the evolution of the two angles when $\varphi$ increases from 0 to $\left.\frac{\pi}{2}\right)$. If $k \geq \sqrt{2}$, all the fibers break at $\varphi=\pi / 4$.

Case of an arbitrary loading. We now consider that both the characteristic angle $\varphi$ and the amplitude of the loading vary. This corresponds to the 


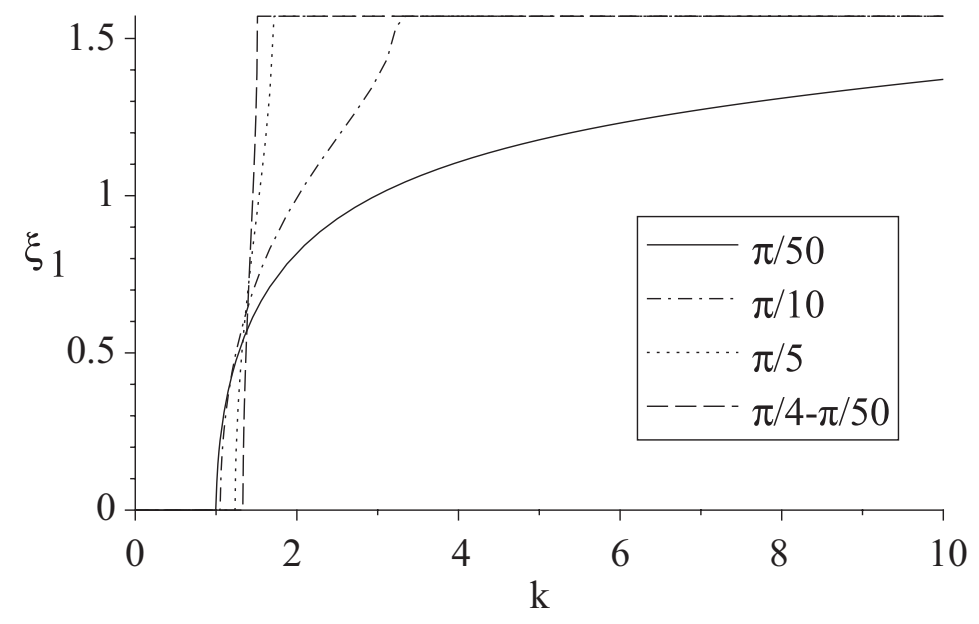

Figure 2: Examples of evolution of the damage variable $\xi_{1}$ for different values of $\varphi$

239 


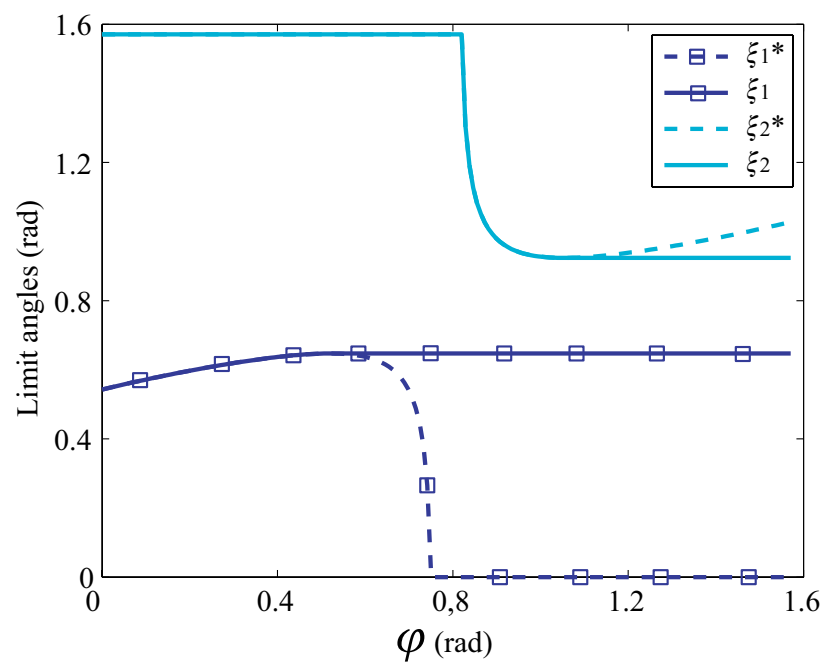

Figure 3: Evolution of the damage variables $\xi_{1}$ and $\xi_{2}$ as functions of $\varphi$ in the case of a circular loading (an increasing $\varphi$ with $k<\sqrt{2}$ constant). $\xi_{1}^{*}$ and $\xi_{2}^{*}$ are the virtual damage variables due to the current loading without taking the loading history into account.

The elasticity range becomes:

$$
\mathcal{S}=\left\{\mathbf{E} / \forall \xi \in A, \mathbf{n}(\xi) \cdot \mathbf{E}(k, \varphi) \cdot \mathbf{n}(\xi)-\varepsilon_{r}<0\right\}
$$

The distortion of the surface is simply the change of the set $A$. More details for the model are described in Brunon (2011).

\section{Influence of the homogenization method on the shape of the stress strain curves}

This section compares the properties of the two homogenization methods when they are applied to the simple fiber breaking damage model defined in the preceding section. All the graphs of this part correspond to the biaxial loading described on Fig.7(a). 


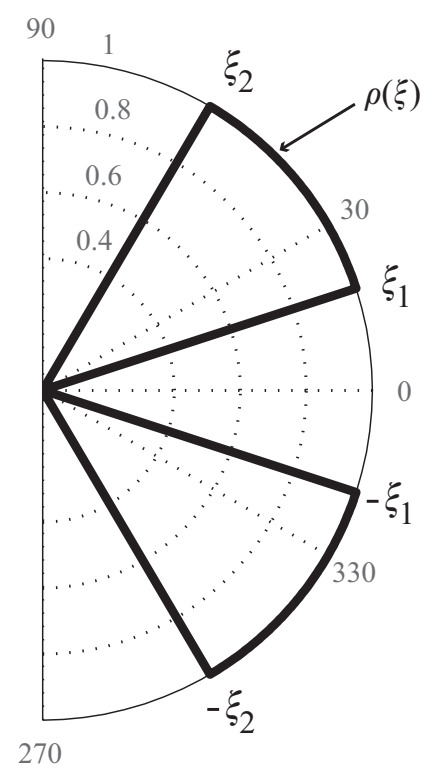

Figure 4: Shape of the density function for a particular damage case $(k=1.5, \varphi=\pi / 10$ and $\pi / 3)$.

\subsection{Macroscopic structure tensor properties}

Let us observe some properties of the resulting macroscopic structure tensor $\mathbf{H}$. We can immediately observe that $H_{12}=H_{21}=0$ because the function $\cos * \sin$ is odd. Indeed, since the integration intervals are always symmetrical with respect to 0 , these two components are always zero, even when damage occurs. Besides, damage affects the components of $\mathbf{H}$ directly. The even nature of functions $\cos ^{2}$ and $\sin ^{2}$ leads to:

$$
\mathbf{H}=\frac{2}{\pi} \int_{0}^{\frac{\pi}{2}} \rho(\xi, \mathbf{D}) \mathbf{n}(\xi) \otimes \mathbf{n}(\xi) \mathrm{d} \xi
$$

An example of the evolution of the nonzero components of $\mathbf{H}$ for a loading up to rupture and for a uniform angular distribution prior to damage, is given 
in Fig.5.

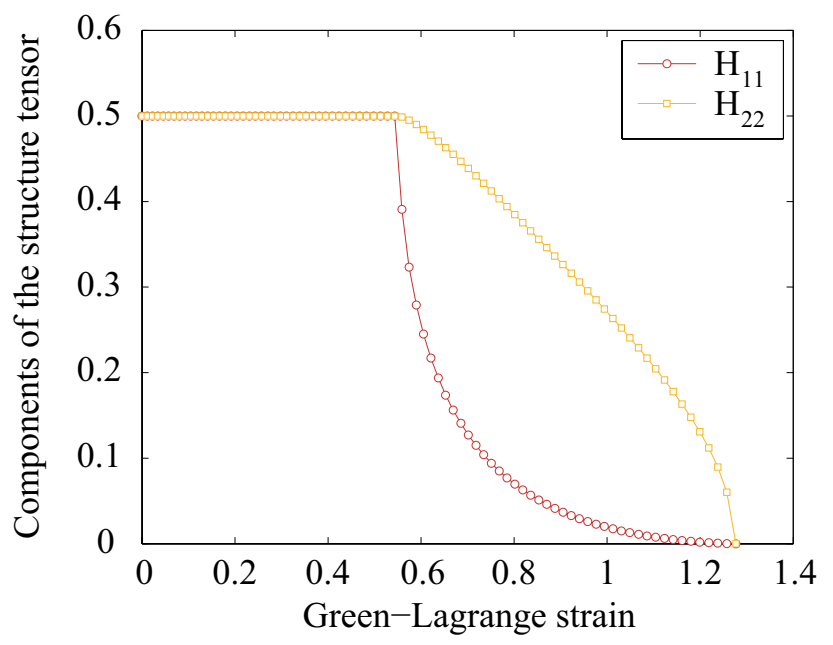

Figure 5: An example of the evolution of the components of the structure tensor $\left(\varepsilon_{r}=0.5\right.$, $\varphi=\pi / 10)$.

264

\subsection{Homogenization methods comparison}

A plot of the fibers' contribution to the strain energy for each method (Fig.6) shows that under the current assumptions of uniform angular distribution prior to damage and brittle linear fibers, the two macroscopic models behave differently when damage occurs. This difference can be observed by expanding the expressions of these energies (Eq.40,41): it comes down to the difference between the integral of a square and the square of an integral. Indeed, for $\xi_{1}$ and $\xi_{2}$ constant (especially prior to damage), the ratio of $\psi_{f}^{K}$ to $\psi_{f}^{E}$ is constant throughout the loading and independent of the value of the elastic parameter $E$. 


$$
\begin{array}{r}
\psi_{f}^{E}=\frac{E}{\pi} \int_{\xi_{1}}^{\xi_{2}}(\mathbf{M}(\xi): \mathbf{E})^{2} \mathrm{~d} \xi=\frac{E}{\pi} \int_{\xi_{1}}^{\xi_{2}}\left(E_{1} \cos ^{2} \xi+E_{2} \sin ^{2} \xi\right)^{2} \mathrm{~d} \xi \\
\psi_{f}^{K}=\frac{E}{2}(\mathbf{H}: \mathbf{E})^{2}=\frac{2 E}{\pi^{2}}\left(\int_{\xi_{1}}^{\xi_{2}}\left(E_{1} \cos ^{2} \xi+E_{2} \sin ^{2} \xi\right) \mathrm{d} \xi\right)^{2}
\end{array}
$$

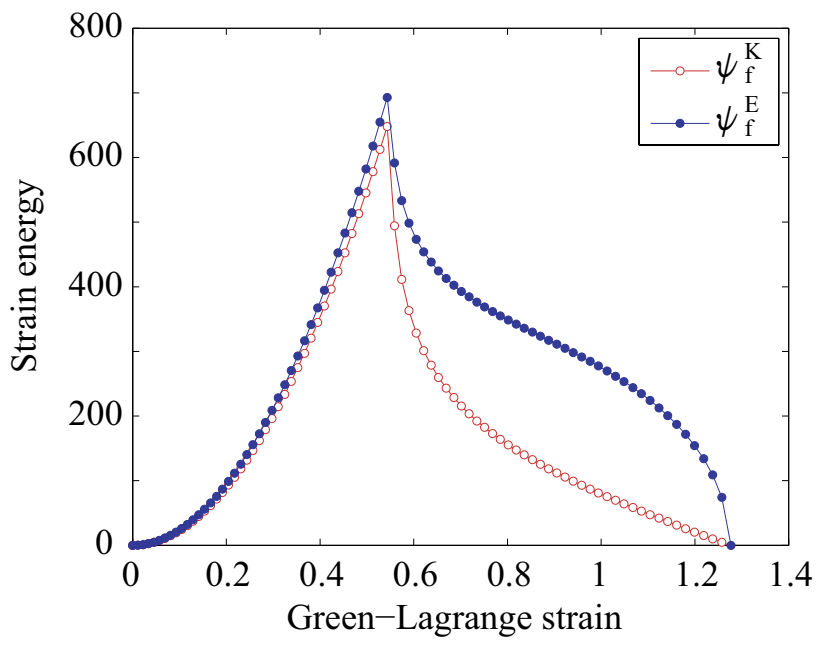

Figure 6: Comparison of the macroscopic strain energies of the fibrous tissue for the two proposed homogenization methods.

Besides, the shape of the components of tensor PK2 (Fig.7, b) show that the $K$ method leads to the same value of the two nonzero components of $\mathbf{S}_{f}^{K}$ prior to damage, whereas the tension applied to the tissue is not equibiaxial. Conversely, with the $E$ method, this unrealistic result is not obtained. This is probably an illustration of the possible non-physical results obtained when the structure tensor theory is applied to a planar isotropic fiber distribution (Holzapfel et al, 2010). However for both homogenization methods, the 
Cauchy stress components (Fig.7, c) are consistent with the components of the strain tensor. Finally, there is a clear difference between the two methods concerning the concavity of the stress component corresponding to the least solicited direction. The softening part of the constitutive relation is much more anisotropic using the $E$ method than using the $K$ method.

\section{Extensions of the model}

In this part, two extensions related to biological tissues are proposed for the damage model and the two homogenization methods are compared for all of them. In the two cases, we consider that the angular distribution of the fibers is uniform before damage.

\subsection{Case of damageable fibers}

The general case described in the previous section is based on the assumption that the fibers are brittle. We now assume that they are damageable. This is a realistic assumption as collagen fibers have a substructure consisting of fibrils which can break progressively (Kastelic et al., 1978).

We choose here a continuous description of the 1D damage in the collagen fibers. The behavior of a fiber is described in Fig.8: we introduce the damage variable $D$, whose evolution is linear, and the damage strain $\varepsilon_{D}$. In the case of a tissue which is initially isotropic and whose fiber density is a binary variable, there is a duality relation between $\rho$ and the damage variable $D$, which enables a simple description of stress homogenization (Eq.43). 


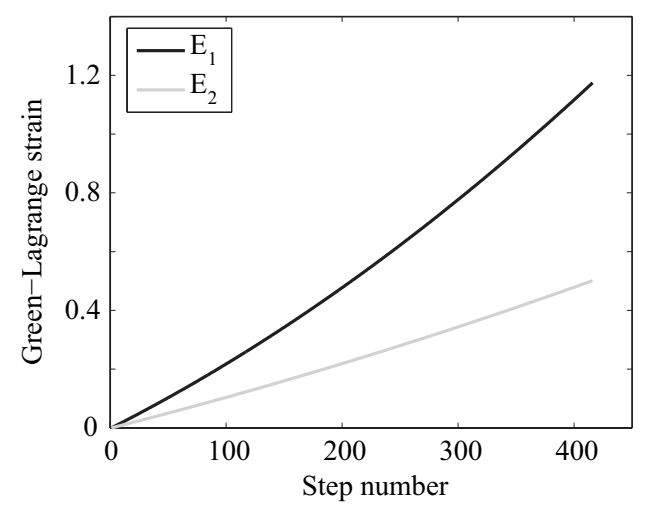

a)

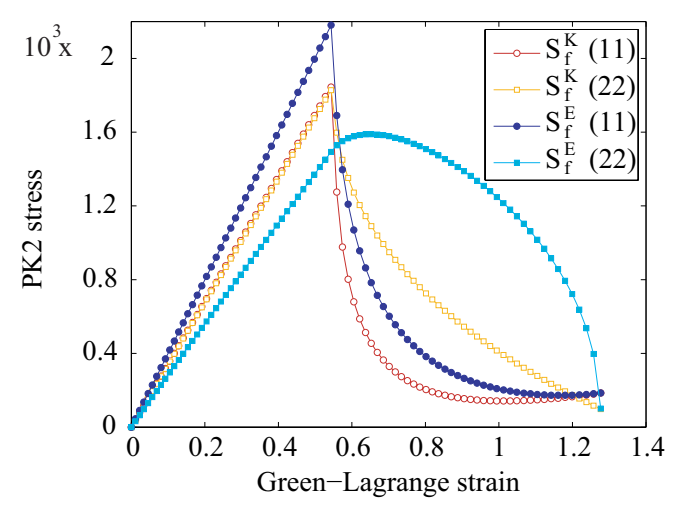

b)

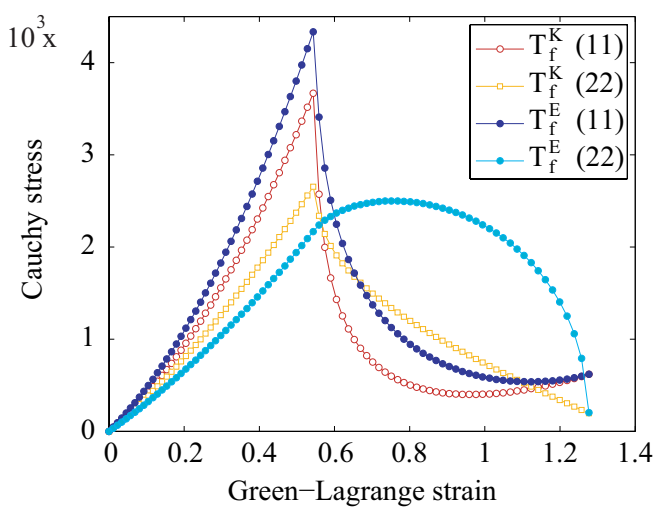

c)

Figure 7: Biaxial strain loading applied to the tissue (a); evolution of the tensor components of PK2 (b) and Cauchy (c) as functions of the loading amplitude. The stress components do not revert back to 0 after complete rupture of the fibers because of the presence of the matrix. 


$$
\begin{aligned}
& E \text { method: }\left\{\begin{array}{l}
\phi_{f}=\frac{1}{2} E(1-D(\boldsymbol{\Gamma}, \xi)) \varepsilon_{f}^{2} \\
\psi_{f}^{E}=\int_{A_{0}} \frac{1}{2} E(1-D)(\mathbf{n} . \mathbf{E} . \mathbf{n})^{2} \mathrm{~d} \xi
\end{array}\right. \\
& K \text { method: }\left\{\begin{array}{l}
\mathbf{H}=\frac{1}{\pi} \int_{A_{0}} \rho(\boldsymbol{\Gamma}, \xi) \mathbf{n} \otimes \mathbf{n d} \xi \\
\psi_{f}^{K}=\frac{1}{2} E(\mathbf{H}: \mathbf{E})^{2}
\end{array}\right.
\end{aligned}
$$

In these expressions, tensor $\boldsymbol{\Gamma}$ represents the value of the strain tensor $\mathbf{E}$ which led to the current macroscopic damage state of the tissue. Since this state is characterized by two independent variables $\xi_{1}$ and $\xi_{2}$, we distinguish ${ }^{1} \boldsymbol{\Gamma}$ and ${ }^{2} \boldsymbol{\Gamma}$ which led to the current values of $\xi_{1}$ and $\xi_{2}$ respectively. The piecewise linear evolution of the variable $D$ or $\rho$ is given within the range $[0, \pi / 4]$ (Eq.44). Within the range $[\pi / 4, \pi / 2]$, the expression is similar, but involves ${ }^{2} \boldsymbol{\Gamma}$.

$$
\begin{gathered}
D= \begin{cases}1 & \text { if } \xi \leq \xi_{1} \\
\frac{\varepsilon_{f}^{*}(\xi)-\varepsilon_{D}}{\varepsilon_{r}-\varepsilon_{D}}=\frac{{ }^{1} \Gamma_{1} \cos ^{2} \xi+{ }^{1} \Gamma_{2} \sin ^{2} \xi-\varepsilon_{D}}{\varepsilon_{r}-\varepsilon_{D}} & \text { if } \xi_{1} \leq \xi \leq \xi_{1}^{\prime} \\
0 & \text { if } \xi_{1}^{\prime} \leq \xi\end{cases} \\
\rho\left(\xi{ }^{1} \boldsymbol{\Gamma}\right)=1-D\left(\xi{ }^{1} \boldsymbol{\Gamma}\right) \forall \xi
\end{gathered}
$$

The shape of the strain energy for each method is given in Fig.9. We obtain a smoothed peak for the evolution of the Cauchy stress in the tissue, corresponding to a more progressive macroscopic damage of the tissue (Fig.10). 


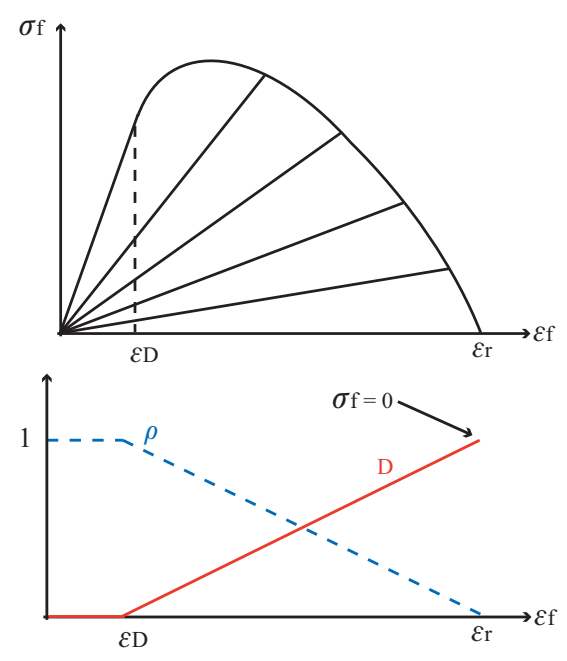

a)

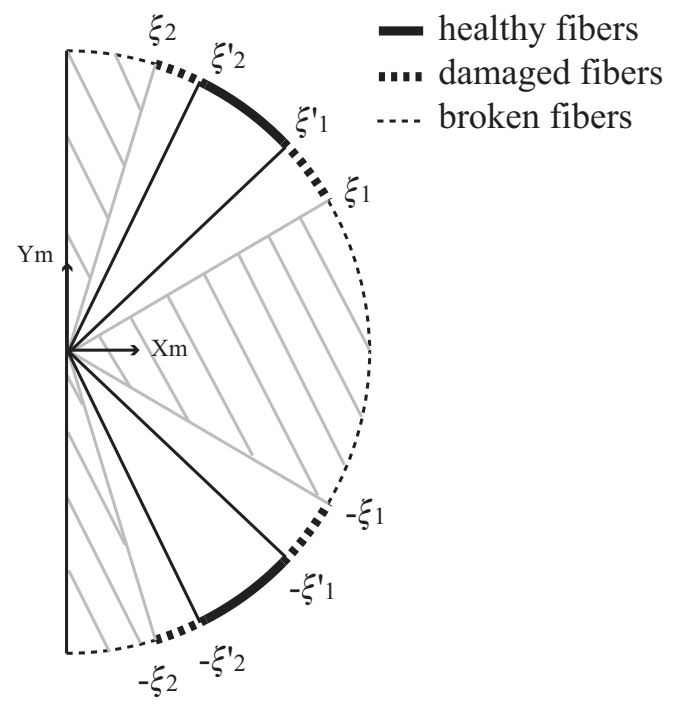

b)

Figure 8: Constitutive law of a damageable fiber and evolution of the damage variable D and density variable $\rho$ throughout the loading (a); damage state of the tissue's fibers (b). 


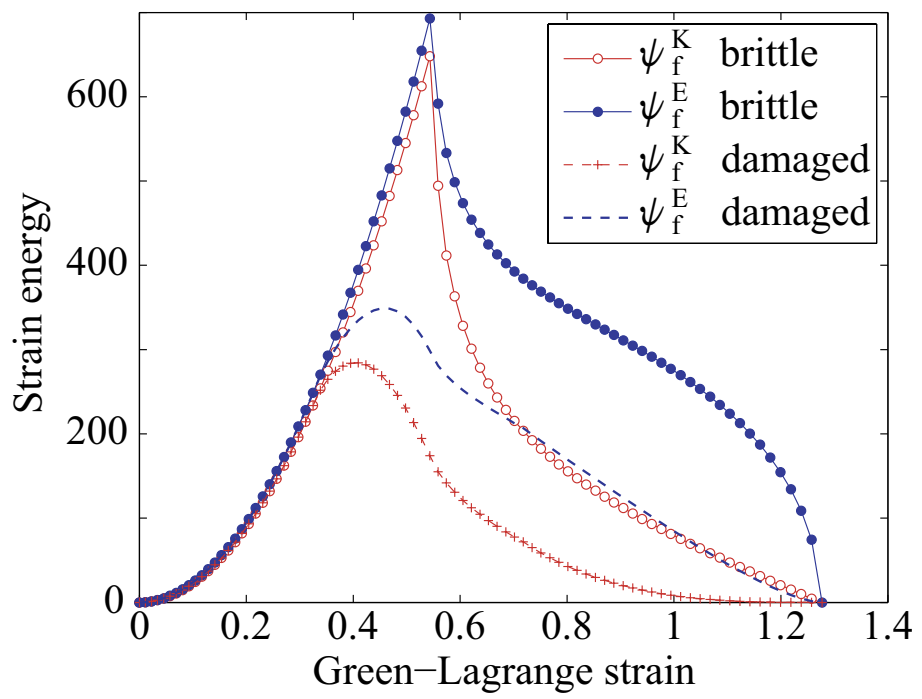

Figure 9: Strain energy during biaxial tension loading for the $K$ and $E$ methods in the case of brittle fibers or damageable fibers. 


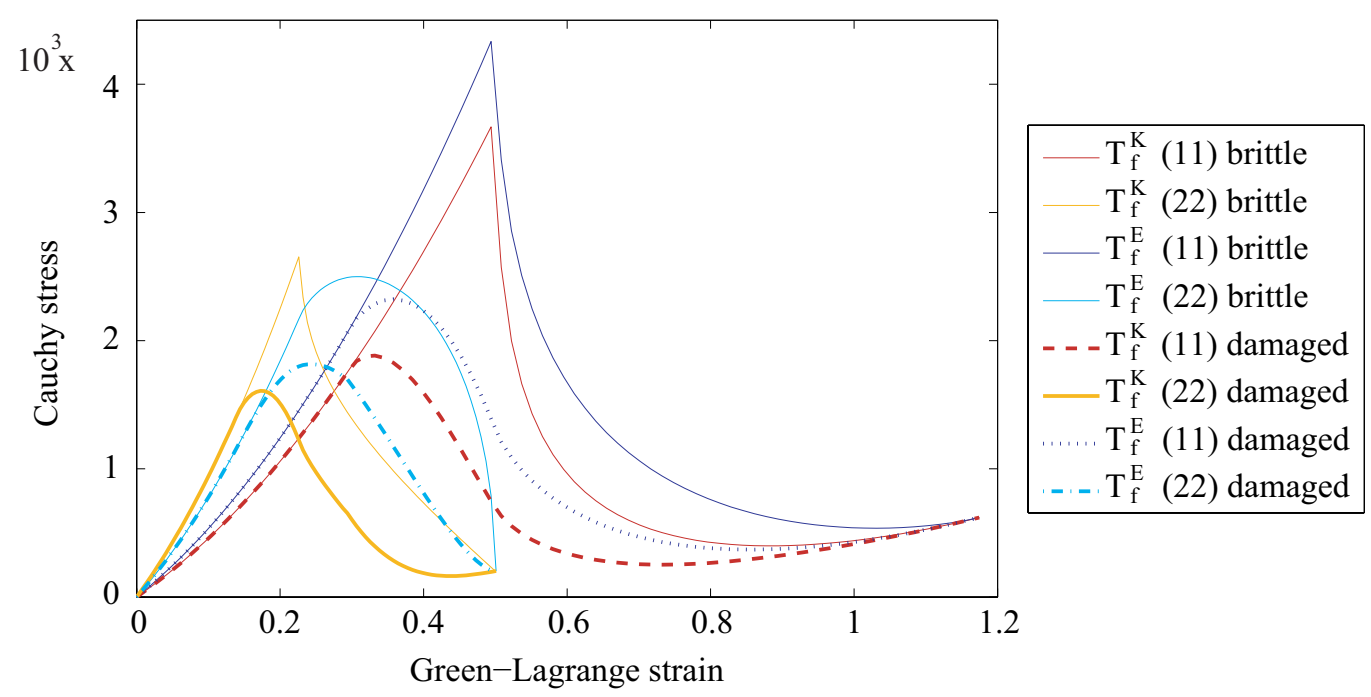

Figure 10: Components of the Cauchy stress tensor of the tissue for the $K$ and $E$ methods in the case of brittle fibers or damageable fibers, as functions of the corresponding components of $\mathbf{E}$.

$$
K \text { method: }\left\{\begin{array}{l}
\mathbf{H}=\frac{1}{\pi} \int_{A_{0}} \rho(\xi) t(\xi) \mathbf{n} \otimes \mathbf{n} \mathrm{d} \xi \\
\psi_{f}^{K}=\frac{1}{2} E(\mathbf{H}: \mathbf{E})^{2}
\end{array}\right.
$$

Fig.12 shows the evolution of the components of the stress tensor: at the beginning of the loading, they are null since no fiber is stretched. The strain in the fibers gradually increases to $\varepsilon_{t}$ and the components of $\mathbf{H}$ increase to their maximum values. Then, rupture including the uncrimping phenomenon in our model leads to an unrealistic shape of the strain energy. Indeed, when the fibers uncrimp, the strain energy increases because both the number of stretched fibers and the amplitude of the loading increase. Then, once all the fibers have uncrimped, the energy continues to increase only because of the loading. Thus, the slope of the energy curve is more gentle once all the fibers 


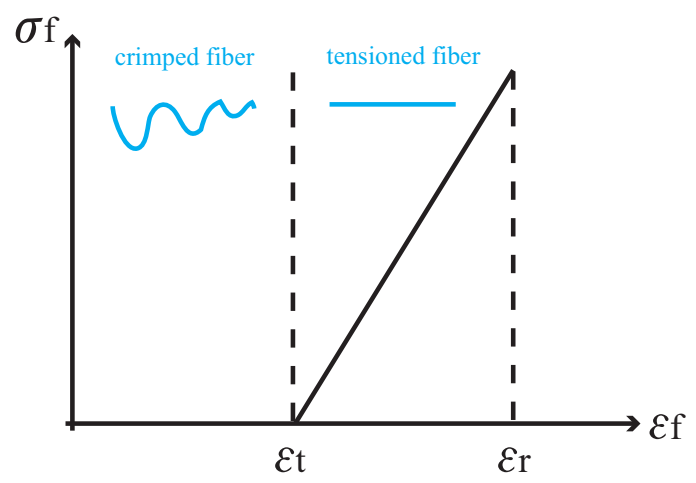

Figure 11: Behavior of a fiber taking uncrimping into account.

are stretched than when some are still crimped. When the fibers uncrimp, the global behavior of the tissue actually "catches up" with the behavior without taking uncrimping into account, leading to steeper slopes of the energy and stress curves. This is a limitation of the model: if all the fibers uncrimp before damage begins, it leads to an energy trend which is never observed experimentally. In order to obtain the "toe region" which is often observed experimentally and which is attributed to the uncrimping of the fibers, only a fraction of the fibers must be stretched when damage begins; this could be obtained using a random distribution of the fibers' crimping, which is not in the scope of this paper as it requires a two-scale computation.

\section{Conclusions}

The objective of this work was to compare the response of two homogenization methods, available in the literature, in the case of damaging fibers within a fibrous membrane. For that purpose we propose a very simple anisotropic damage model able to account for several phenomena observed 

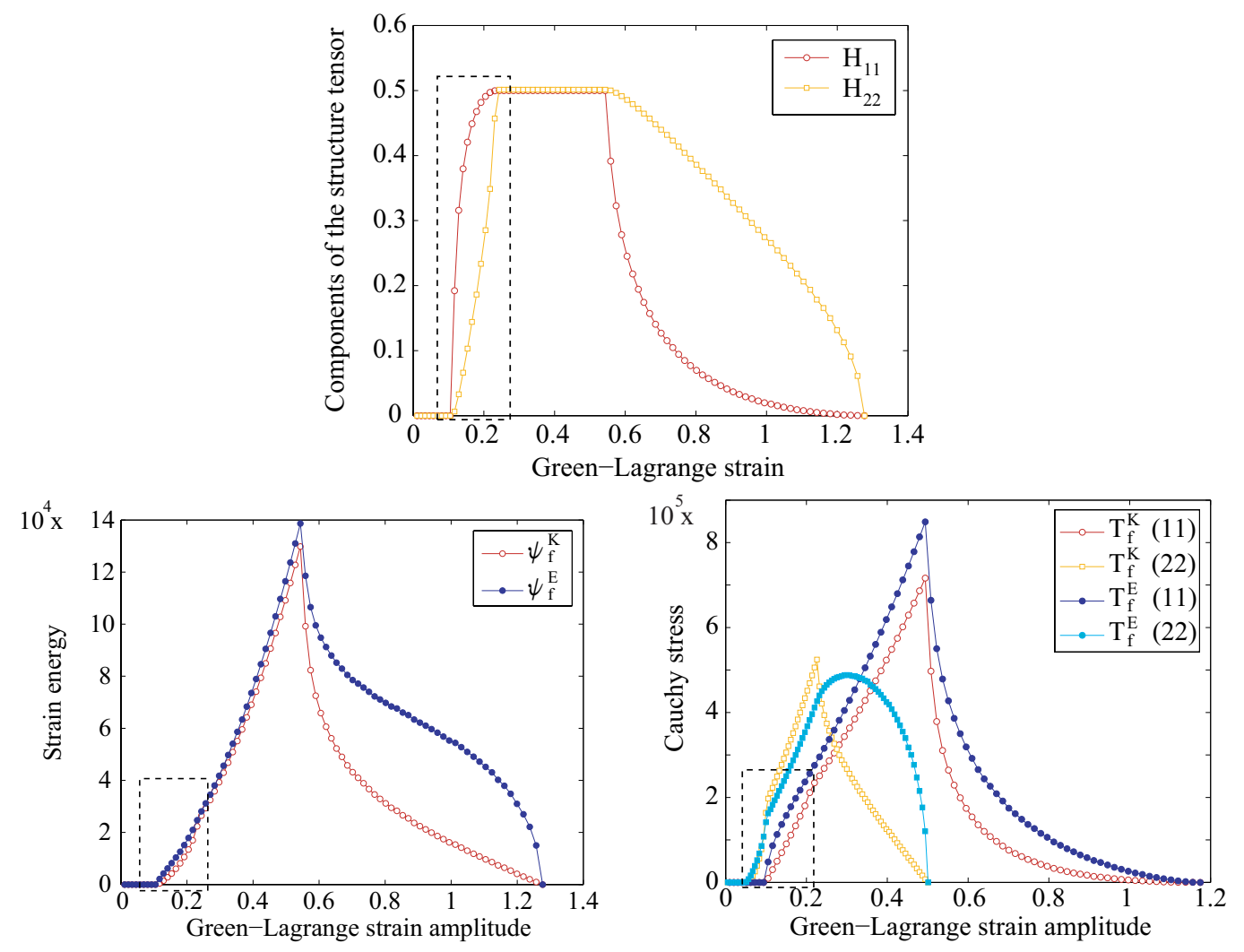

Figure 12: Evolution of the structure tensor, the strain energy and the components of the Cauchy tensor under biaxial tension for initially crimped fibers $\left(\varepsilon_{t}=0.15, \varepsilon_{r}=0.5\right)$. The box emphasizes the area where the obtained behavior is not realistic. 
experimentally.

The evolution of macroscopic damage based on fiber fracture at the microscopic scale is described using two scalar variables. The explicit expression of these variables as functions of the material parameters and of the macroscopic strain loading enables a rapid implementation of the fracture behavior law of the tissue. Besides, this paper does not deal with any phenomenon which would require reverting to the microscopic scale.

The main limitation of this model is that it considers the tissue's damage to be borne by the fibers alone independently of the components of the tissue (namely the matrix) with the assumption that the fibers are isolated from one another. This leads to a drastic nature of the constitutive law, that could be tone down by introducing fiber interactions, a distribution of the fibers properties or a possible decohesion between the fibers and the matrix.

The implementation of the two homogenization methods enabled us to compare them: in the case of an isotropic tissue, the difference between the two methods appears during damage and is due to the different formulation of the tissue's macroscopic strain energy. The mathematical expression of this difference makes us believe that a heterogeneous angular distribution of the fibers (for an initially anisotropic tissue) would reveal a disparity between the two methods right from the elastic zone. Another fundamental difference is that the $K$ method cannot take into account the behavior on the fibers' scale because the constitutive law is applied directly to the macroscopic tissue.

Depending on the homogenization method, the principle of the identification of the damage model is different: indeed, the $E$ method based on the strain energy of a fiber requires knowing the behavior and fracture strain 
of a fiber; these parameters can be determined independently of the fibrous tissue (see, for example, (Sasaki and Odajima, 1996; Svensson et al., 2010) for collagen fibers). Nevertheless, while the response of the fibrous tissue can be determined a priori, the response of the matrix is still to be identified. In the case of the $K$ method, the constitutive law is applied to the tissue rather than to the fibers; consequently, the identification of the macroscopic parameters of the tissue is required both for the fibrous tissue and for the matrix; only the fracture strain of the fibers can be determined experimentally a priori. In practice, the identification of material parameters associated with damage requires being able to control the evolution of damage so that rupture does not to occur too rapidly. This constitutes the main difficulty of the experimental characterization of damage.

The model proposed in this study can be applied to initially isotropic membrane tissues. Therefore, the prospective applications of this study concern the identification of the model's parameters in practical cases. In the case of biological tissues, the fracture modeling of the liver capsule could enhance a model of the human body and improve the prediction of liver injuries during an impact.

\section{Appendix A: Details about the description of damage}

The objective of this development is to define, for a radial loading with an amplitude greater than the bounds defined in Eq.(33), the angular sectors in which the fibers break. This corresponds to the resolution of $\varepsilon_{f}-\varepsilon_{r}>0$. In the general case, since $\sin ^{2} \xi=1-\cos ^{2} \xi$ : 


$$
\begin{aligned}
& \cos \varphi \cos ^{2} \xi+\sin \varphi\left(1-\cos ^{2} \xi\right)>\frac{1}{k} \\
& \cos ^{2} \xi(\cos \varphi-\sin \varphi)>\frac{1-k \sin \varphi}{k}
\end{aligned}
$$

Two cases need to be distinguished:

$$
\begin{gathered}
\varphi \in\left[0, \frac{\pi}{4}\right] \Rightarrow \cos \varphi-\sin \varphi \geq 0 \Rightarrow \cos ^{2} \xi>\frac{1-k \sin \varphi}{k(\cos \varphi-\sin \varphi)} \\
\varphi \in\left[\frac{\pi}{4}, \frac{\pi}{2}\right] \Rightarrow \cos \varphi-\sin \varphi \leq 0 \Rightarrow \cos ^{2} \xi<\frac{1-k \sin \varphi}{k(\cos \varphi-\sin \varphi)}
\end{gathered}
$$

394 In the following, only the case $\varphi \in\left[0, \frac{\pi}{4}\right]$ is explained.

These equations hold provided that the right-hand side is positive, i.e.:

$$
1-k \sin \varphi \geq 0 \Leftrightarrow k \leq \frac{1}{\sin \varphi}
$$

Therefore, one can write:

$$
\cos \xi>\sqrt{\frac{1-k \sin \varphi}{k(\cos \varphi-\sin \varphi)}} \forall k \in\left[\frac{1}{\cos \varphi}, \frac{1}{\sin \varphi}\right]
$$

$$
\forall k \in\left[\frac{1}{\cos \varphi}, \frac{1}{\sin \varphi}\right]: \cos \xi \geq \sqrt{\frac{1-k \sin \varphi}{k(\cos \varphi-\sin \varphi)}}
$$




$$
\begin{aligned}
\varphi \in\left[0, \frac{\pi}{4}\right]:\left\{\begin{array}{l}
\xi \leq \arccos \sqrt{\frac{1-k \sin \varphi}{k(\cos \varphi-\sin \varphi)}} \\
\text { ou } \\
\xi \geq-\arccos \sqrt{\frac{1-k \sin \varphi}{k(\cos \varphi-\sin \varphi)}}
\end{array}\right. \\
\varphi \in\left[\frac{\pi}{4}, \frac{\pi}{2}\right]:\left\{\begin{array}{l}
\xi \geq \arccos \sqrt{\frac{1-k \sin \varphi}{k(\cos \varphi-\sin \varphi)}} \\
\text { ou } \\
\xi \leq-\arccos \sqrt{\frac{1-k \sin \varphi}{k(\cos \varphi-\sin \varphi)}}
\end{array}\right.
\end{aligned}
$$

Acknowledgement 1. The authors would like to thank the Region RhôneAlpes for its financial support.

\section{References}

Balzani D, Schröder J, Gross D, 2006. Simulation of discontinuous damage incorporating residual stresses in circumferentially overstretched atherosclerotic arteries. Acta Biomaterialia 2 (6), 609-618.

Brunon A, 2011. Characterization and modeling of the hepatic tissues failure. Ph.D. thesis, Institut National des Sciences Appliquées de Lyon.

Cacho F, Elbischger P, Rodriguez J, Doblare M, Holzapfel G, 2007. A constitutive model for fibrous tissues considering collagen fiber crimp. International Journal of Non-Linear Mechanics 42 (2), 391-402. 
Calvo B, Pena E, Martinez M, Doblare M, 2007. An uncoupled directional damage model for fiberd biological soft tissues. Formulation and computational aspects. International Journal for Numerical Methods in Engineering 69 (10), 2036-2057.

Gasser T, Holzapfel G, 2006. Modeling the propagation of arterial dissection. European Journal of Mechanics-A/Solids 25 (4), 617-633.

Gasser T, Ogden R, Holzapfel G, 2006. Hyperelastic modelling of arterial layers with distributed collagen fiber orientations. Journal of the royal society interface 3 (6), 15-35.

Holzapfel G, 2000. Nonlinear solid mechanics. Wiley New York.

Holzapfel G, Ogden R, 2010. Constitutive modelling of arteries. Proceedings of the Royal Society A: Mathematical, Physical and Engineering Science 446 (2118):1551-1597.

Hurschler C, Loitz-Ramage B, Vanderby Jr R, 1997. A structurally based stress-stretch relationship for tendon and ligament. Journal of biomechanical engineering 119 (4), 392-399.

Kastelic J, Galeski A, Baer E, 1978. The multicomposite structure of tendon. Connective tissue research 6 (1), 11-23.

Kuhl E, Garikipati K, Arruda E, Grosh K, 2005. Remodeling of biological tissue: Mechanically induced reorientation of a transversely isotropic chain network. Journal of the Mechanics and Physics of Solids 53 (7), 1552-1573. 
Lanir Y, 1983. Constitutive equations for fibrous connective tissues. Journal of Biomechanics 16:1-12.

Liao H, Belkoff S, 1999. A failure model for ligaments. Journal of biomechanics $32(2), 183-188$.

Menzel A, Waffenschmidt T, 2009. A microsphere-based remodelling formulation for anisotropic biological tissues. Philosophical Transactions of The Royal Society A: Mathematical, Physical and Engineering Sciences 367 (1902): 3499-3523.

Rodríguez J, Cacho F, Bea J, Doblaré M, 2006. A stochastic-structurally based three dimensional finite-strain damage model for fibrous soft tissue. Journal of the Mechanics and Physics of Solids 54 (4), 864-886.

Sacks M, Gloeckner D, 1999. Quantification of the fiber architecture and biaxial mechanical behavior of porcine intestinal submucosa. Journal of biomedical materials research 46 (1), 1-10.

Sáez P, Alastrué V, Peña E, Doblaré M, Martínez M, 2012. Anisotropic microsphere-based approach to damage in soft fibered tissue. Biomechanics and Modeling in Mechanobiology 11 (5):595-608.

Sasaki N, Odajima S, 1996. Elongation mechanism of collagen fibrils and force-strain relations of tendon at each level of structural hierarchy. Journal of biomechanics 29 (9), 1131-1136.

Svensson R, Hassenkam T, Hansen P, Peter-Magnusson S, 2010. Viscoelastic behavior of discrete human collagen fibrils. Journal of the Mechanical Behavior of Biomedical Materials 3 (1), 112-115. 